\title{
New challenges in biorobotics: Incorporating soft tissue into control systems
}

\author{
Barry A. Trimmer \\ Biology Department and Biomimetic Devices Laboratory, Tufts University, Medford, MA, USA
}

(Received 1 August 2008; final version received 12 November 2008)

\begin{abstract}
The development of truly biomimetic robots requires that soft materials be incorporated into the mechanical design and also used as an integral part of the motor control system. One approach to this challenge is to identify how soft animals control their movements and then apply the found principles in robotic applications. Here I show an example of how a combination of animal kinematics, neural patterning and constitutive modelling of tissues can be used to explore motor control in the caterpillar, Manduca sexta. Although still in the early stages, these findings are being used to design and fabricate a new type of robot that does not have a rigid skeleton and is structured entirely from soft or compliant materials. It is hoped that this new robotic platform will promote the development of actuators, sensors and electronics that are compatible with soft materials.
\end{abstract}

Keywords: neuromechanics; caterpillar; locomotion; muscles; soft-bodied robots

\section{Introduction}

A major challenge in the field of biorobotics is to reproduce the astoundingly precise, robust and versatile motions of animals. In fact, most robots can be instantly recognised as synthetic machines because they fail to negotiate complex environments as smoothly and quickly as their biological counterparts. One factor that helps animals adapt movements so well to a changing environment is their reliance on soft and flexible materials. In contrast most robots are built from very stiff components that have more predictable mechanical performance but lack compliance and 'self-correction'. The extensive use of soft materials in robot construction is limited by the difficulty of integrating non-linear materials into traditional control systems. One goal of the research described here is to begin to understand how soft tissues contribute to the control of animal movement and use these findings to build conformable robots from soft materials.

Current highly flexible (hyper-redundant) biologically inspired machines are mostly built from concatenated rigid modules with multi-axis joints between them. Well-known examples include the 'snakelike' robots of Hirose (1993), Chirikjian and Burdick (1995), Borenstein (Granosik et al. 2005), Choset (Wolf et al. 2003) and Miller (Miller 2002). Similar modular designs have been used as reconformable machines (Yim 1994) and form the basis for many undulating or swimming robots (Crespi et al. 2005; Wilbur et al. 2002).

However, most flexible animals are soft-bodied with no rigid skeleton at all. Instead they use highly compliant ma- terials and vary their stiffness using hydraulics, muscle tension and tissue compaction. Of these soft-bodied animals, caterpillars are the most successful climbing herbivores on the planet. Their multi-legged crawling is distinct from the bouncing gaits of articulated animals (Farley et al. 1993; Full and Farley 2000) and the peristaltic movements of worms or molluscs (Trimmer and Issberner 2007). Caterpillars use passive grip to secure themselves to complex branched substrates (Mezoff et al. 2004) and have a multidimensional workspace, able to bend, twist and crumple in ways that are not possible with a rigid skeleton. They use dynamic hydrostatics to vary body tension and can cantilever over gaps that are $90 \%$ the length of their bodies. Remarkably, during the lifetime of a caterpillar there are no major changes in the number or arrangement of muscles, and the crawling gait does not change despite a 10,000 -fold increase in mass. A robot that captures the essential control system of a caterpillar could be highly scalable.

There have been very few attempts to build truly softbodied robots with the intrinsic capacity to deform, twist and crawl. Several ingenious flexible designs have been developed based on peristalsis (Mangan et al. 2002, Menciassi and Dario 2003, Saga and Nakamura 2004), conformable wheels (Sugiyama et al. 2005) and continuously bending elements ('continuum robots'; Hannan and Walker 2003, Walker et al. 2005). Each of these has its own advantages, but most only function in a specific environment, and none of them can climb or completely collapse to access restricted spaces.

\footnotetext{
*Email: barry.trimmer@tufts.edu
} 
The present work describes locomotion in the caterpil$\operatorname{lar}$ (Manduca sexta) and some of the neuromechanics that appear important for its movements. In particular, the elastomeric nature of its muscles is altered by neural activation in complex but predictable ways. It is our hypothesis that by controlling the material state of tissues such as muscle, soft animals can greatly simplify neural encoding of hyperflexible movements. These findings are being applied to the design and construction of a new type of collapsible, soft-bodied robot ('Softbot').

\section{Manduca as a model system for distributed control of movements}

Manduca caterpillars are an excellent model system for studying the neuromechanics of soft-bodied movements. Their movements are achieved through the coordination of concatenated segments (Figure 1 ) each containing $\approx 70$ distinct muscles. Because Manduca larvae metamorphose into adult moths having jointed limbs, their muscles are organised into individual, discrete units each with distinct orientations and attachments analogous to the muscular organisation of vertebrates (Figure 2A). Despite the complexity of Manduca's movements and the large number of muscles, each muscle is innervated by a single motoneuron (or occasionally two motoneurons), and there are no inhibitory motor units. Therefore, most of Manduca's movements are controlled by a few hundred motoneurons whose activity can be monitored using electrodes implanted in the muscles of freely moving animals. In addition, Manduca's locomotion contrasts markedly with most model systems. Caterpillars are extraordinarily successful climbers and can burrow (in preparation for pupation) and hold on to the substrate using a very effective passive grasping system (Mezoff et al. 2004).

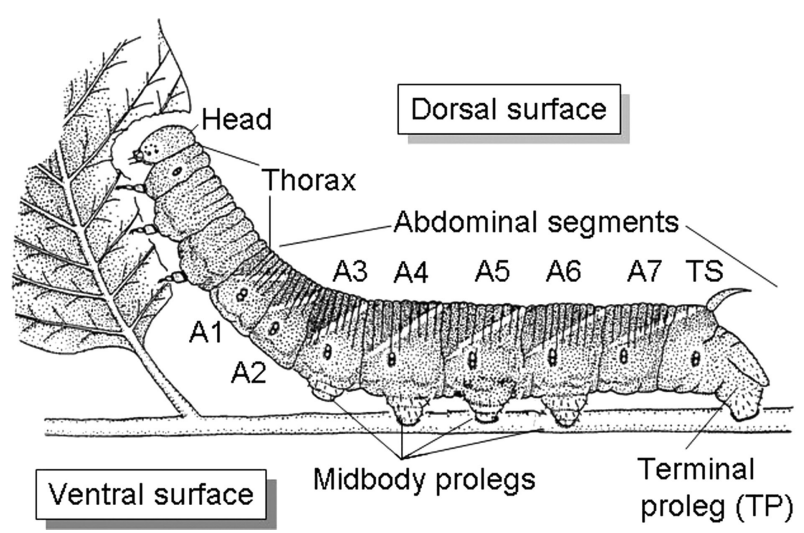

Figure 1. External anatomy of the caterpillar, Manduca sexta, which is an excellent model system for understanding how soft animals control their movements. The abdominal segments are labelled A1-A7 with the most posterior segment designated the TS. The main gripping appendages are the prolegs found on A3A6 and a specialised pair on the TS.
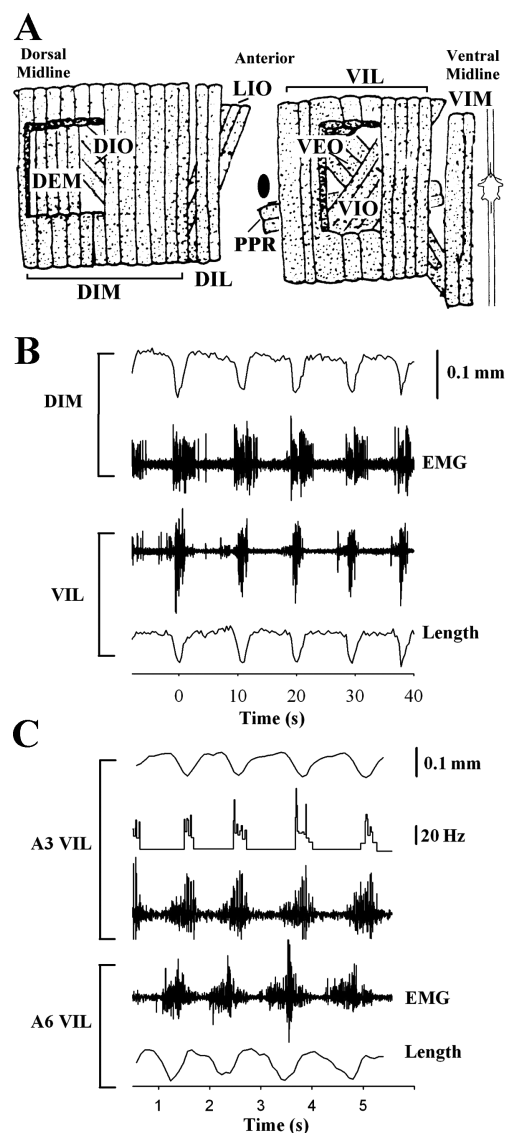

Figure 2. (A) Major muscles on one side of an abdominal segment (from Levine and Truman 1985). Muscle names are identified in the text. (B) Strain cycles of two muscles in segment A4 (DIM, dorsal: top; VIL, ventral: bottom) and corresponding EMGs during horizontal crawling. Dorsal and ventral muscles are phase-locked and co-active, although DIM continues to be activated during its re-extension. (C) Strain cycles of VIL in two segments (A3, A6) and their corresponding EMGs during horizontal crawling. Also shown in A3 is the instantaneous spike frequency of the motoneuron driving VIL, derived from the EMG (see text). The EMG and VIL strain in A3 are phase-delayed relative to A6 but co-active for much of their duty cycle. The interactions between muscle placements, material properties and neural commands are being used to guide the design and control of soft robots.

\section{Caterpillar neuromechanics}

\section{Kinematics}

A three-dimensional kinematic study of straight-line crawling shows that caterpillars do not move by wormlike peristalsis (Trimmer and Issberner 2007). Waves of movement pass from the terminal segment (TS; Figure 1) towards the head, and there is a transition in the kinematics between posterior segments and those in the midbody. The TS and adjacent abdominal segment (A7) are lifted and pulled forward into stance phase; the segments then pivot around the terminal proleg (TP) attachment point in a motion that 
resembles an inverted pendulum. Vertical displacements precede changes in horizontal velocity by $30^{\circ}$ (one step $=$ $360^{\circ}$ ). In the midbody segments the horizontal velocity and height are essentially in phase (lead or lag $<10^{\circ}$ ), and each body segment is at its maximum length during the stance phase. As the wave moves forward, segments compress in the first part of the swing phase and re-extend before entering stance again (Trimmer and Issberner 2007).

The dorsal and ventral parts of each segment change length in phase with one another, implying that lifting and bending across the length of the caterpillar occurs by folding of the intersegmental membranes. Unexpectedly, the length and radius of each body segment co-vary; that is, each segment is the narrowest when it is the shortest. Hence, unlike the leech (Skierczynski et al. 1996, Wilson et al. 1996), segment volume in Manduca is not necessarily conserved during a crawl; so tissue, fluid or air (in the tracheal system) can be transported from one part of the body to another and back again.

The essential kinematics of crawling are not different on curved or flat surfaces, although there are slight changes in the relative timing and duration of movements in some parts of the body. These subtle changes occur in de-brained larvae, suggesting that some of Manduca's adaptability is mediated by local biomechanical or proprioceptive events. Remarkably, all the major kinematic features of horizontal crawling are identical during vertical climbing (Griethuijsen and Trimmer), suggesting that the crochet gripping system is an extraordinarily effective attachment mechanism. This insensitivity to orientation is also a desirable feature for any climbing robot based on Manduca locomotion.

\section{Motor patterns during horizontal crawling}

In contrast with the widely accepted model of caterpillar locomotion (Barth 1937; Holst 1934, 1943; Snodgrass 1961), both dorsal and ventral muscles in each body segment co-contract (Figure 2B). Single muscles (e.g. ventral internal lateral muscle, VIL) in different segments are phase-delayed, but even muscles that are four segments apart are co-active for $70 \%$ of their duty cycle (Figure 2C). Some muscles (e.g. the dorsal internal muscle, DIM) continue to be activated as they re-extend (Figure 2B), suggesting that they play an important role in resisting stretch, perhaps stiffening the body wall to transmit forces. Muscles therefore act as a reconfigurable skeletal in addition to their role as motors. This finding has important consequences for the design of soft robots because it highlights the need to integrate functions (chassis, engine) that are kept separate in traditional machines. At least one major muscle (DIO) is not always active during crawling but instead is responsible for bracing and casting behaviours, which implies that subsets of muscles can be used for different activities. An extremely important feature of Manduca as a model system is that, by using direct motoneuron stimulation in reduced prepa- rations, we can identify characteristic features of the extracellular muscle potentials and translate the EMGs of intact animals into motoneuron spike frequencies (Figure 2C). This technical breakthrough allows us to catalogue the neural command sequences of individual motoneurons in the overall motor patterns. These sequences carry central commands to the periphery, and their results can be visualised in the kinematics. (We effectively know what the muscles are 'told' and what movements result.) The force contribution of any single muscle in response to these commands can also be measured by replaying natural spike trains into isolated muscles while they are undergoing natural strain cycling (see the subsection 'Reconstructed crawling work loops'). This combination of single-cell command resolution and single muscle kinematics and dynamics makes Manduca a unique model for understanding soft body control.

\section{Passive and active muscle properties}

Studies on the characteristics of VIL show that it has a large tetanus-to-twitch force ratio (8.5; see Rheuben and Kammer 1980), a peak isotonic contraction velocity (and amplitude) at $10 \%$ of the peak active tension and a relatively slow contraction (peak tension in one second) (Woods et al. 2008). In contrast to most arthropod striated muscles, VIL has an enormous working strain range (50-100\%), presumably enabling large body deformations even in the absence of stiff levers. During sinusoidal strain cycling, VIL dissipates work (clockwise work loops) in both passive (unstimulated), and tetanic states, with higher stiffness and more work dissipation during stimulation (Woods et al. 2008). This property is similar to that of the locust flight muscle (Josephson et al. 2000), except that VIL operates at 10 times the strain and 30 times more slowly. Furthermore, when VIL is cycled at increasing speeds, the work loops change trajectory (viscoelasticity), and passive dissipation becomes a larger proportion of the total work absorbed during stimulation. These time-dependent non-linear properties will strongly impact passive dynamic responses during active contraction. Muscle can be viewed as a soft elastomeric material and can be characterised using large deformation continuum mechanical theory (Fung 1993). Using this approach we have made empirical stress-strain measurements and developed constitutive models for both passive and active muscles and for the strain-rate dependency of their properties (Dorfmann et al. 2007, 2008). These equations can be implemented in dynamics simulation models to more fully describe how Manduca controls its movements.

\section{Reconstructed crawling work loops}

Length changes measured in VIL during crawling (e.g. Figure 2C) were used to construct simplified strain ramps that were applied to VIL. VIL was also stimulated with 


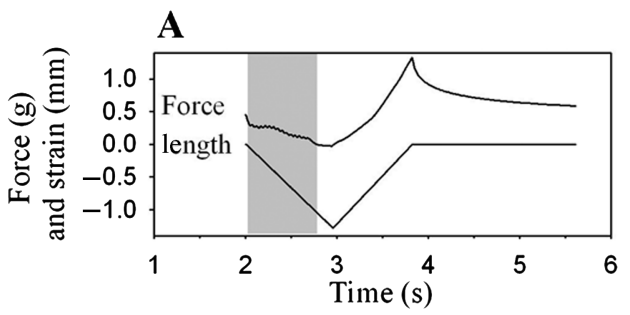

B
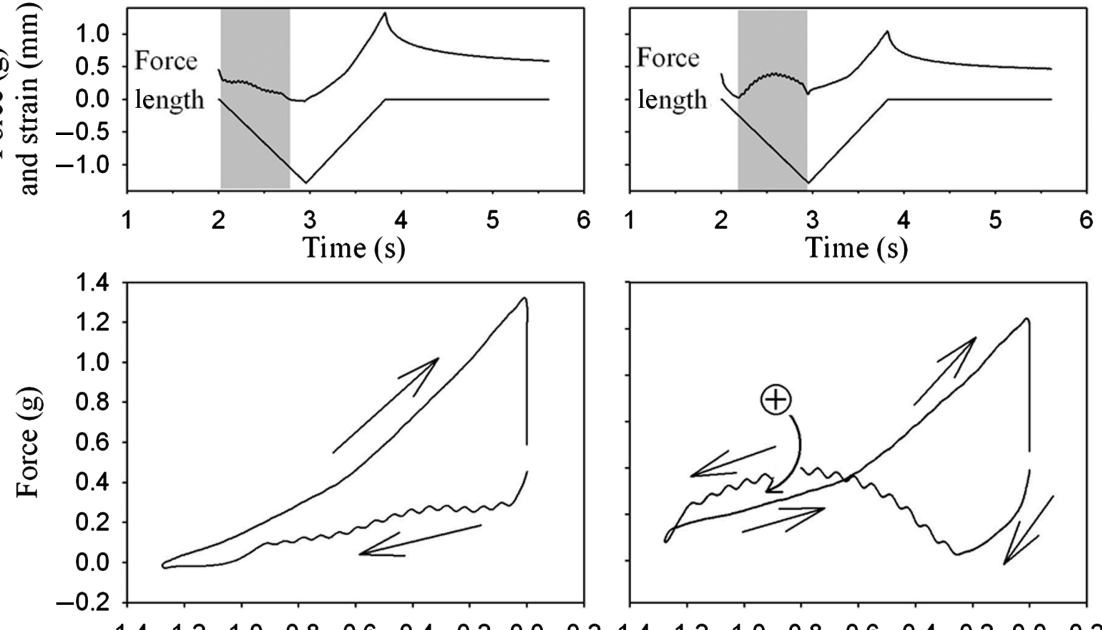

$-1.4-1.2-1.0-0.8-0.6-0.4-0.2 \quad 0.0 \quad 0.2-1.4-1.2-1.0-0.8-0.6-0.4-0.2 \quad 0.0 \quad 0.2$

Length $(\mathrm{mm})$

Length $(\mathrm{mm})$

Figure 3. Effect of stimulus timing on simulated crawling work loops for VIL. Both traces and work loops are from a single muscle undergoing a simplified crawling strain cycle with a stimulus $(20 \mathrm{~Hz}$, grey bars) lasting $0.68 \mathrm{~s}$, beginning at the start of shortening (A) or after a delay of $0.19 \mathrm{~s}$, which normally occurs during crawling (B). Within each cycle VIL acts as a motor when it is shorter (+ indicates a region of positive work) and a break or damper (dissipating mechanical work) when it is longer. This is an example of a muscle performing two distinct functions within each strain cycle.

spike trains whose intensity, duration and timing matched those occurring during crawling (Figure 2C, trace 2). These 'crawling work loops' show that timing of a natural stimulus has a major impact on VIL performance. Phase-advanced (Figure 3A), or phase-delayed (not shown), stimuli resulted in work dissipation throughout the strain cycle. Stimulus timing that matched crawling produced a region of positive work output (actuation; Figure 3B). Interestingly, the region of positive work was strongly dependent on the stimulus duration (Figure 4), suggesting that muscle function could be controlled by small variations in motor neuron activity (Woods et al. 2008). It is expected that these highly

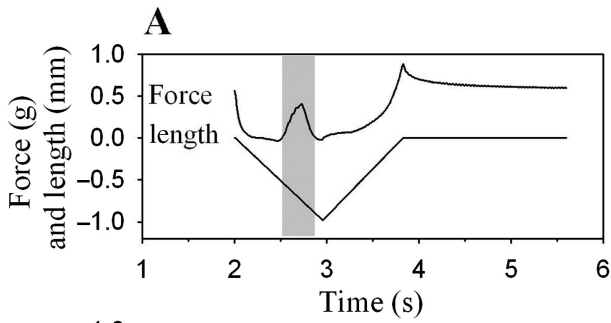

B
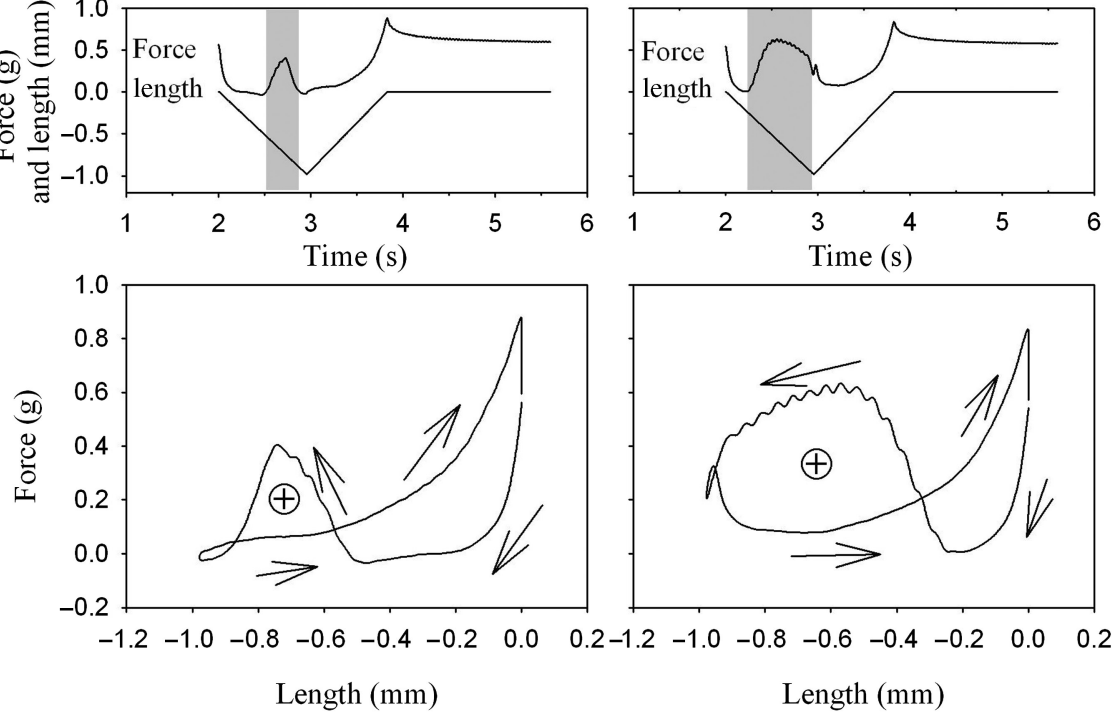

Figure 4. Effect of stimulus duration on simulated crawling work loops for VIL. Both traces and work loops are from a single muscle (from a different animal than that in Figure 2) undergoing a simplified crawling strain cycle with a stimulus (20 Hz, grey bars) ending at the peak of shortening. (A) Stimulus duration $=0.28 \mathrm{~s}$; (B) stimulus duration $=0.68 \mathrm{~s}$; + indicates a region of positive work (actuation). 
non-linear features of the motor system are exploited to generate adaptable and robust control. The complexity of the muscle response is actually a rich source of peripheral computation ('morphological computation'; Pfeifer 2000, Pfeifer and Iida 2005). Rather than minimising such complexity, soft robots should take advantage of non-linear material properties and thereby simplify and speed up the central command process.

\section{Elastomeric properties of Manduca muscle}

The fundamental pseuodoelastic properties of passive and tetanically stimulated muscle have been characterised using constant velocity strain changes. The dissipation of work by VIL resembles that of high carbon particle rubber (Dorfmann and Ogden 2004), including its tendency to soften (Mullins effect). These properties can be accurately modelled using constitutive elastomeric theory (Dorfmann et al. 2007). In addition, Manduca muscles are viscoelastic over a variety of strain rates (Dorfmann et al. 2008) including slow increases in residual stress at low strains, velocitydependent yield after stretch, changes in work loop trajectories at different strain cycle frequencies and stress hysteresis at constant velocity strains (Woods et al. 2008). These properties could be mechanisms for autonomous control of the biomechanical responses, thereby lowering the demand for real-time neural control. It is also possible that neural commands exploit some of these complex material states to more effectively control hyper-redundant locomotion. These questions are being explored in simulation models (the 'Virtual Manduca') and hardware robotic environments ('Softbot').

\section{Robot design concept ('Softbot')}

The robot under development (Figure 5) is a contoured cylinder constructed from highly elastic silicone rubber. It moves using shape memory alloy (SMA) coils as actuators, bonded directly with the inside of the body wall. Instead of circular and longitudinal 'muscles' used in most wormlike designs, Softbot has discrete groups of actuators modelled on those of the caterpillar. Future prototypes will have a set of passive-grip/active-release opposable legs capable of gripping flat surfaces, irregular objects and wires or ropes. The body contains an inner compartment (the 'gut') that will be used to hold components of the control system and additional payload. The space between the 'gut' and body wall can be pressurised to transmit forces and regulate stiffness. Release of pressure will also allow the robot to collapse and compress into a free-form volume limited only by the payload size. Softbot is expected to be fault tolerant (Haroun Mahdavi and Bentley 2003), capable of extreme mobility (including shape changing) and exceedingly simple to build.

\section{Actuators}

The essential features of Manduca muscles can be produced using SMA coils from a single wire wound to provide different strains and forces (Kim et al. 2005, Menciassi et al. 2004, Tsakiris et al. 2005). For many robot designs SMAs are considered rather slow, but thin wire SMAs can actuate and recover in less than one second, closely matching the performance of caterpillar muscles. The SMA is activated by resistive heating using a low-voltage power source. Although the current can be regulated to vary the rate and force of contraction we have chosen to use trains of pulses that are frequency modulated to achieve better control (Ayers 2002). This approach also mimics the frequency encoding system used in animal motor systems (see the subsection 'Controller hardware'). The prototype uses $150-\mu \mathrm{m}$ nitinol which normally has a working strain of $3 \%$ and a recovery force of $3 \mathrm{~N}$. When wound as a micro-spring $1 \mathrm{~mm}$ in diameter, the actuator works over $100 \%$ strain and develops $0.3 \mathrm{~N}$ of repeatable working force (Figure 6). These SMA coils are bonded with the body wall whose elasticity serves as a bias (recoil) spring.

\section{Body wall}

The main body of Softbot is cast from a soft silicone elastomer (Dragonskin ${ }^{\mathrm{TM}}$, Smooth-On, Inc.). As part of

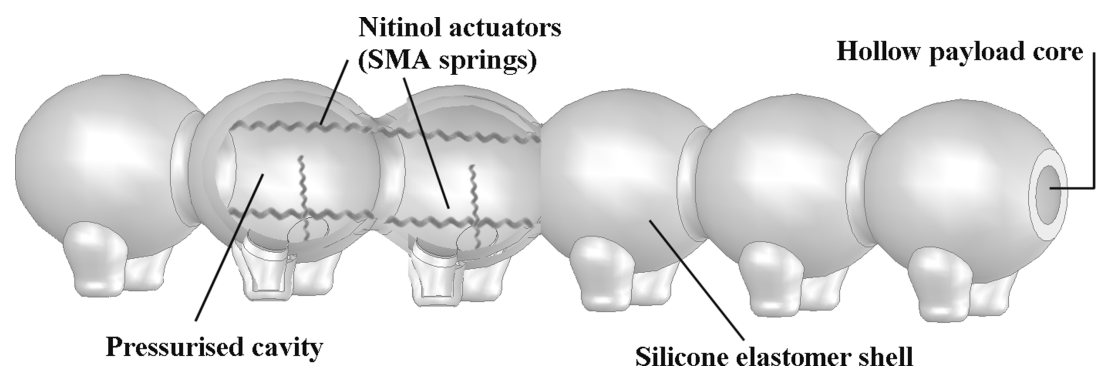

Figure 5. General bodyform of Softbot in partial cutaway longitudinal section. The design is a simplified modular version of the abdomen of Manduca sexta. The SMA coils analogous to muscles are bonded with the body wall spanning the segment boundaries. 
A

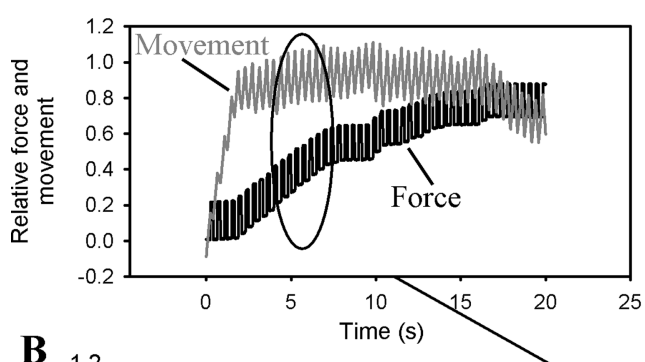

$2.4 \mathrm{~Hz}, 40 \%(\mathrm{ON})$

duty cycle

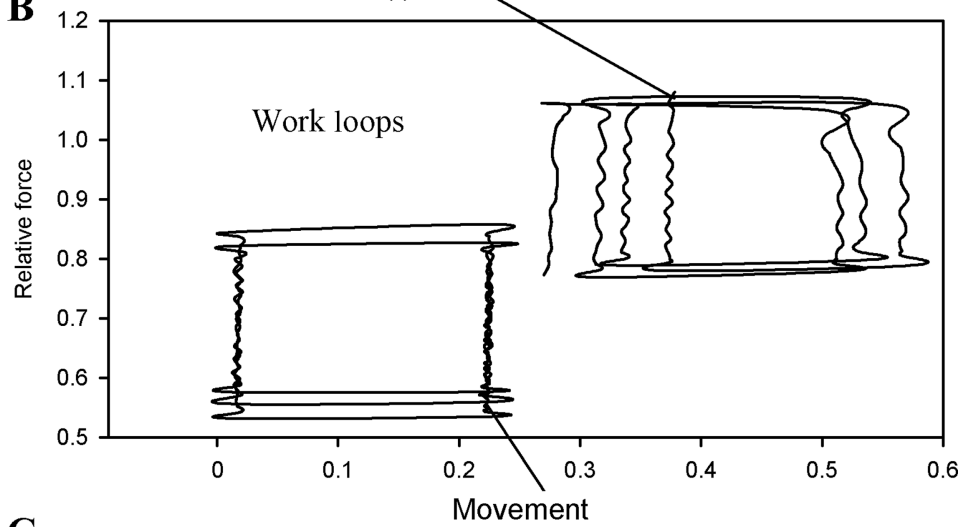

C

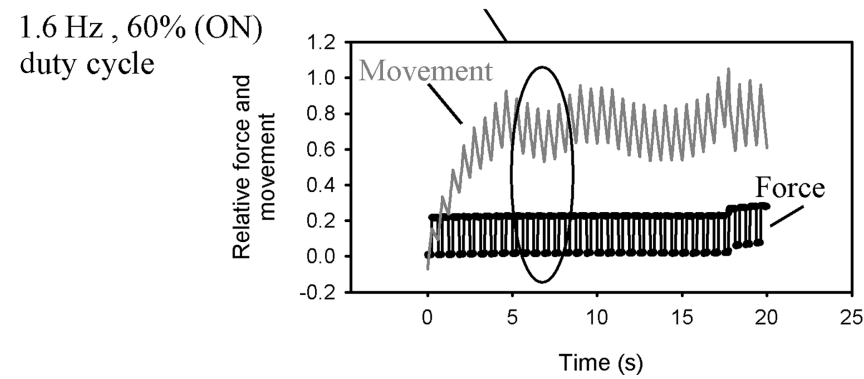

Figure 6. SMA spring actuators can be operated in different work loop configurations, using pulsed current at different frequencies and duty cycles. The performance of a 1-mm diameter spring is shown carrying a 30 -g load activated for $20 \mathrm{~s}$ at either $2.4 \mathrm{~Hz}$, $40 \%$ duty cycle (A) or $1.6 \mathrm{~Hz}, 60 \%$ duty cycle (C). Work loops plotted from the indicated response region of each trial are shown in (B). The upper line (red) tracks the force, and the lower line (black) is the movement of the actuator, both plotted on a relative scale. The spring develops $0.3-1 \mathrm{~N}$ force and exhibits strains of $150-200 \%$.

the design of the robot we have developed a process to quickly manufacture a large number of prototypes for testing changes in morphology and material properties. The method uses rapid prototyping tools to produce casting shells with the SMAs mounted in place. The silicone body wall is thickened and contoured in segments to resemble the caterpillar and promote useful deformations. This shape will eventually be optimised using structurally based constitutive models of the caterpillar and the robot. Recent studies on the caterpillar body wall show that it is anisotropic (twice as stiff circumferentially compared to axially; Huai-Ti et al. 2008). This feature can designed into the Softbot body wall, using oriented fibers or by varying the wall thickness.

\section{Controller hardware}

Each actuator is controlled using a pulsed current source driven by a master oscillator to maintain the overall cadence of a crawl. This rhythm is coupled to a second oscillator whose frequency and duty cycle can be varied to generate square wave bursts which are analogous to the trains of action potentials that cause muscle contraction. Alterations in these parameters can be used to control the overall amplitude of the force and contraction of the actuator or to move the work-loop responses into a different operating range (Figure 6). This method of driving the actuators has been found to be more consistent than direct amplitude modulation (e.g. changing the driving current; Ayers 2002), and it has the additional advantage of a rich and complex range 


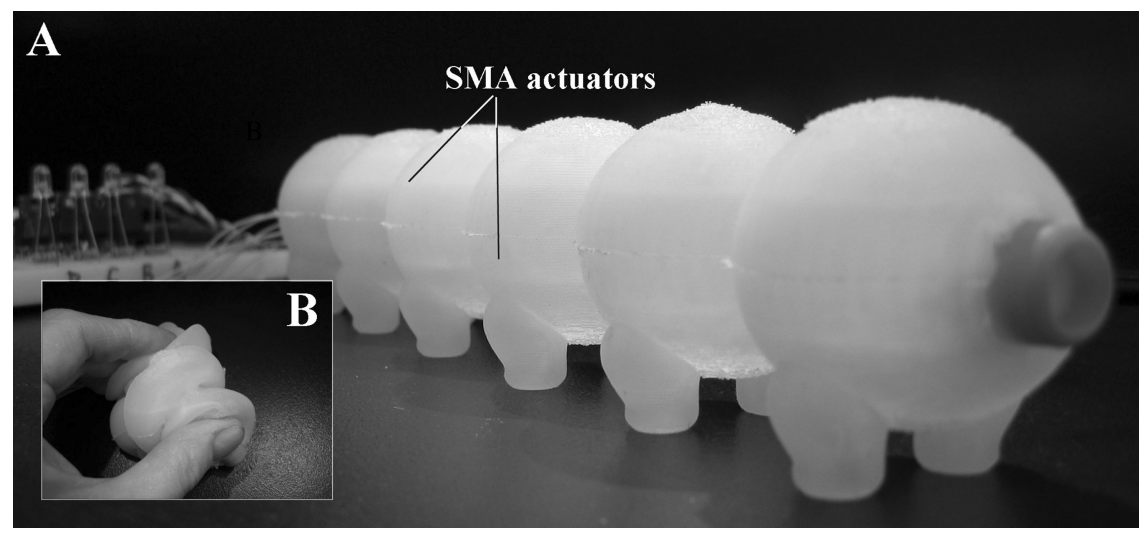

Figure 7. The prototype Softbot. (A) The robot is inflated, and the positions of two actuator SMA springs inside the body shell are indicated. (B) Shows the robot collapsed and folded.

of responses. The coupled signal can be set globally or differently for each actuator so that the phase of the pulses relative to one another, their polarity (inverted pulse timing analogous to an inhibitory neuron connection), frequency and duty cycle are all variables available for movement control.

Currently the robot is tethered to the offboard power supply, and the hardware is implemented at the level of discrete components driven by LabVIEW programs. This system controls 48 SMAs but can be readily expanded to 72 simultaneous outputs. The eventual goal is to make Softbot autonomous, using VLSI circuits and flexible PCBs housed inside the 'gut'.

\section{Softbot assembly and control}

The current prototype is $24 \mathrm{~cm}$ long, $4 \mathrm{~cm}$ in diameter and contains only 12 actuators arranged in two serial rows on opposite sides of the body wall (Figures 5 and 7). Each actuator is capable of generating substantial strain to bend and fold the local body wall. By activating pairs of SMA coils in turn, waves of contraction pass along the body in a simulated crawling motion. Tension in the body wall itself is sufficient to restore coils to their pre-activated length, although adjacent coils can be activated to speed this process (Kim et al. 2005). This first functioning prototype demonstrates that SMA coils and silicone elastomer can be bonded firmly and produce muscle-like movements. A second generation prototype is currently under construction using SMA coils oriented to mimic the main locomotory muscles of the Manduca caterpillar. Because of the mechanical unpredictability of complex soft structures the command sequences for Softbot movements are being discovered and optimised using genetic algorithms (Rieffel et al. 2008). This involves refining a pool of command parameters through simulations of the robot movements in a physics simulator. Various methods are being explored to speed this evolutionary process, including automatic preselection of structural parameters. Robustness is ensured using a multi-component fitness function based on realworld tasks for the robot.

\section{Concluding remarks}

The details of how soft-bodied animals position their bodies appropriately to accomplish complex movements are currently unknown. Despite their extreme flexibility (with enormous degrees of freedom) and lack of discrete joints (to define position or orientation), soft-bodied animals do not have larger or more intricate brains than their rigid skeleton counterparts. The work described here has begun to explore how such flexible movements are controlled by a relatively simple nervous system. The tobacco hornworm (Manduca sexta) offers several powerful advantages for analysing interactions between neural and biomechanical elements. In addition, its muscular organisation is analogous to that of animals with jointed skeletons; so many of the found principles can be applied to the movements of legged vertebrates and adult arthropods, as well as animals with hydrostatic skeletons. By integrating data and methodologies from neuroscience, materials science and robotics we will begin to form a deeper understanding of the role of soft materials in animal locomotion and discover ways to use these materials to produce more versatile and lifelike devices.

\section{Acknowledgments}

This work was partially supported by NSF/IBN grant no. 0117135 to BAT and by the Keck Foundation. The author would like to thank Dr Luis Dorfmann, Dr William Woods and Michael Simon for helpful discussions.

\section{References}

Ayers J. 2002. A conservative biomimetic control architecture for autonomous underwater robots. In: Neurotechnology for biomimetic robots. Cambridge (MA):MIT Press. p. 211-259.

Barth R. 1937. Muskulatur und Bewegungsart der Raupen. Zool Jb Physiol. 62:507-566. 
Chirikjian GS, Burdick JW. 1995. The kinematics of hyperredundant robotic locomotion. IEEE Trans Rob Automat. 11(6):781-793.

Crespi A, Badertscher A, Guignard A, Ijspeert AJ. 2005. AmphiBot I: an amphibious snake-like robot. Rob Auton Syst. 50(4):163-175.

Dorfmann A, Ogden R. 2004. A constitutive model for the Mullins effect with permanent set in particle-reinforced rubber. Int J Solid Struct. 41(7):1855-1878.

Dorfmann A, Trimmer BA, Woods, Jr, WA. 2007. A constitutive model for muscle properties in a soft bodied arthropod. J R Soc Interface. 4:257-269.

Dorfmann A, Woods Jn WA, Trimmer BA. 2008. Muscle performance in a soft-bodied terrestrial crawler: constitutive modelling of strain-rate dependency. J R Soc Interface. 5(20):349362.

Farley CT, Glasheen J, McMahon TA. 1993. Running springs: speed and animal size. J Exp Biol. 185:71-86.

Full RJ, Farley CT. 2000. Musculoskeletal dynamics in rhythmic systems: a comparative approach to legged locomotion. In: Biomechanics \& neural control of posture \& movement. New York: Springer. p. 192-205.

Fung YC. 1993. Biomechanics: mechanical properties of living tissues. 2nd ed. Springer.

Granosik G, Hansen MG, Borenstein J. 2005. The OmniTread serpentine robot for industrial inspection and surveillance. Int J Ind Robots. IR32-2: 139-148.

Griethuijsen L, Trimmer BA. Kinematics of horizontal and vertical caterpillar crawling. J Exp Biol.

Hannan MW, Walker ID. 2003. Kinematics and the implementation of an elephant's trunk manipulator and other continuum style robots. J Robot Syst. 20(2):45-63.

Haroun Mahdavi S, Bentley PJ. An evolutionary approach to damage recovery of robot motion with muscles. In: Banzhaf W, Christaller T, Dittrich P, JKim JT, Zieglre, J editors. Proceedings of the European Conference on Artificial Life (ECAL 2003). Berlin:Springer-Verlag.

Hirose S. 1993. Biologically inspired robots: snake-like locomotors and manipulators. Oxford (New York): Oxford University Press.

Holst EV. 1934. Motorische und tonische Erregung und ihr Bahnenverlauf bei Lepidopteranlarven. Z Vgl Physiol. 21:395414.

Holst EV. 1943. Uber relativ Koordination bei Arthropoden. Pflugers Arch Physiol. 246:847-865.

Huai-Ti L, Dorfmann L, Trimmer B. 2008. Soft cuticle biomechanics: a constitutive model of anisotropy for caterpillar integument. J Theor Biol. Doi 10.1016/j.jtbi.2008.10.018.

Josephson RK, Malamud JG, Stokes DR. 2000. Asynchronous muscle: a primer. J Exp Biol. 203(Pt 18):2713-2722.

Kim B, Lee S, Park JH, Jong-Oh P. 2005. Design and fabrication of a locomotive mechanism for capsule-type endoscopes using shape memory alloys (SMAs). IEEE/ASME Trans Mechatron. 10(1):77-86.

Levine RB, Truman JW. 1985. Dendritic reorganization of abdominal motoneurons during metamorphosis of the moth, Manduca sexta. J Neurosci. 5(9):2424-2431.

Mangan EV, Kingsley DA, Quinn RD, Chiel HJ. Development of a peristaltic endoscope. In: International Congress on Robotics and Automation. p. 347-352

Menciassi A, Dario P. 2003. Bio-inspired solutions for locomotion in the gastrointestinal tract: background and perspectives. Philos Trans A Math Phys Eng Sci. 361(1811):2287-2298.

Menciassi A, Gorini S, Pernorio G, Dario P. A SMA Actuated Artificial Earthworm. In: Proceedings of the 2004 IEEE In- ternational Conference on Robotics and Automation (ICRA 2004). New Orleans.

Mezoff S, Papastathis N, Takesian A, Trimmer BA. 2004. The biomechanical and neural control of hydrostatic limb movements in Manduca sexta. J Exp Biol. 207(Pt 17):3043-3053.

Miller G. 2002. Snake robots for search and rescue. In: Neurotechnology for biomimetic robots. Cambridge (MA): MIT Press. p. $271-284$

Pfeifer R. 2000. On the role of morphology and materials in adaptive behavior. In: From animals to animats. Cambridge, MA: MIT Press. p. 23-32.

Pfeifer R, Iida F. 2005. Morphological computation: connecting body, brain and environment. Jap Scientific Monthly. 58(2):48-54.

Rheuben MB, Kammer AE. 1980. Comparison of slow larval and fast adult muscle innervated by the same motor neurone. $\mathrm{J}$ Exp Biol. 84:103-118.

Rieffel J, Trimmer B, Lipson H. Mechanism as mind: what tensegrities and caterpillars can teach us about soft robotics. In: Bullock JNS, Watson R, Bedau MA, editors. Artificial life XI: Proceedings of the Eleventh International Conference on the Simulation and Synthesis of Living Systems, Winchester, UK. Cambridge, MA:MIT Press.

Saga N, Nakamura T. 2004. Development of a peristaltic crawling robot using magnetic fluid on the basis of the locomotion mechanism of the earthworm. Smart Mat Struct. 13(3):566.

Skierczynski BA, Wilson RJ, Kristan, Jr, WB, Skalak R. 1996. A model of the hydrostatic skeleton of the leech. J Theor Biol. 181(4):329-342.

Snodgrass RE. 1961. The caterpillar and the butterfly. Smithsonian Misc Collec. 143(6):51.

Sugiyama Y, Shiotsu A, Yamanaka M, Hirai S. Circular/spherical robots for crawling and jumping. In: IEEE International Conference on Robotics and Automation, Barcelona.

Trimmer BA, Issberner JI. 2007. Kinematics of soft-bodied, legged locomotion in Manduca sexta larvae. Biol Bull. 212:130-142.

Tsakiris DP, Sfakiotakis M, Menciassi A, La Spina G, Dario P. 2005. Polychaete-like undulatory robotic locomotion. In: Proceedings of the 2005 IEEE International Conference on Robotics and Automation (ICRA 2005); 2005 April 18-22, Barcelona, Spain.

Walker ID, Dawson D, Flash T, Grasso F, Hanlon R, Hochner B, Kier WM, Pagano C, Rahn CD, Zhang Q. 2005. Continuum robot arms inspired by cephalopods. In: Proceedings of the 2005 SPIE Conference on Unmanned Ground Vehicle Technology IV; Orlando, FL.

Wilbur C, Vorus W, Cao Y, Currie S. 2002. A lamprey-based undulatory vehicle. In: Neurotechnology for biomimetic robots. Cambridge, MA: MIT Press.

Wilson RJ, Skierczynski BA, Blackwood S, Skalak R, Kristan, Jr, WB. 1996. Mapping motor neurone activity to overt behaviour in the leech: internal pressures produced during locomotion. J Exp Biol. 199(Pt 6): 1415-1428.

Wolf A, Ben Brown, Jr, H, Casciola R, Costa A, Schwerin M, Shammas E, Choset H. 2003. A mobile hyper redundant mechanism for search and rescue rasks. In: International Conference on Intelligent Robots and Systems; 2003 October, Las Vegas. Las Vegas: IEEE/RSJ.

Woods WA, Jn., Fusillo S, Trimmer BA. 2008. Dynamic properties of a locomotory muscle of the tobacco hornworm Manduca sexta during strain cycling and simulated crawling. J Exp Biol. 211:873-882.

Yim M. Locomotion with a unit modular reconfigurable robot [dissertation]. [Palo Alto (CA)]: Stanford University. 

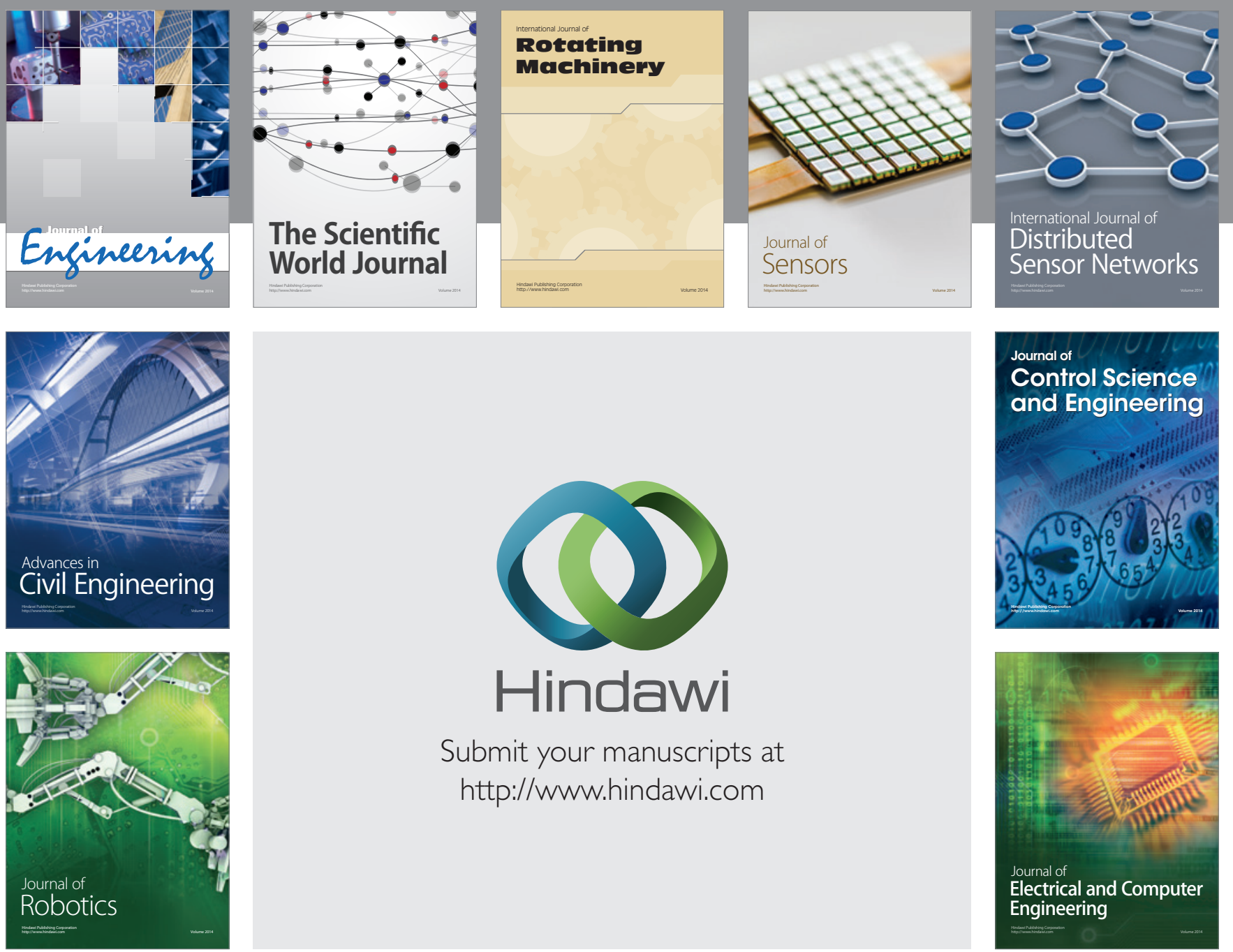

Submit your manuscripts at

http://www.hindawi.com
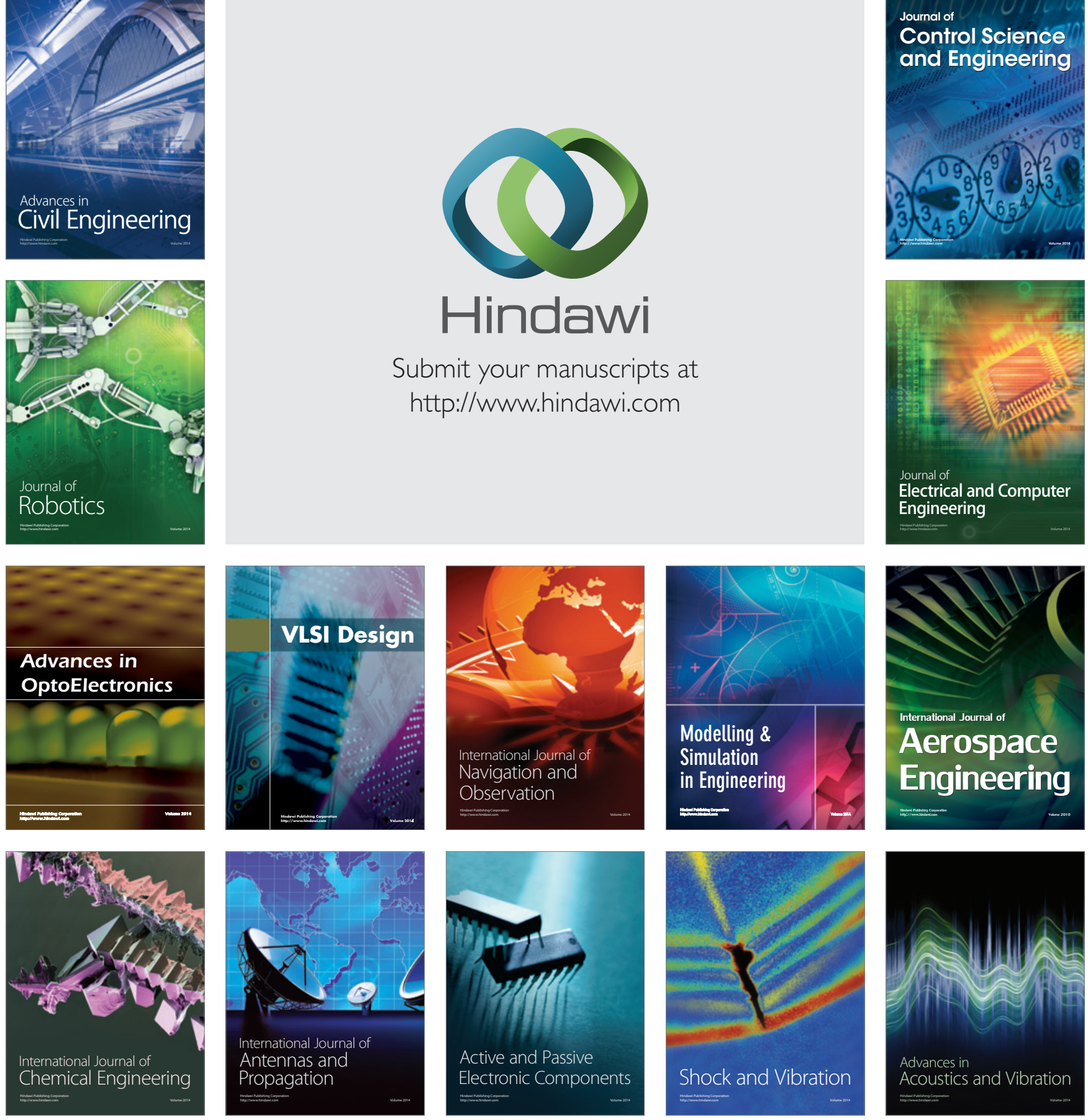